\title{
VALIDATION OF ULTRASOUND FOR INTUSSUSCEPTION AT PEDIATRIC EMERGENCY DEPARTMENT
}

\author{
Y.J. Chang, J.L. Huang, C.T. Wu, D.C. Yan, H.C. Chao, M.S. Kong \\ Chang Gung Memorial Hospital, Taoyuan, Taiwan R.O.C.
}

Background and aims: Intussusception is the one of the most common acute abdomen in children. Ultrasound is being increasingly used the diagnosis of intussuscption. Ultrasound was performed by gastroenterologists at our hospital but not available at night and holidays. The ultrasound training was conducted for pediatric emergency physician. We analyzed our experience to evaluate the validation of ultrasound performed by pediatric emergency physician for the diagnosis of intussusception at emergency department .

Methods: From July 2004 to July 2008 retrospective study was conducted with children suspected intussusception when gastroenterologists were not available. Patients were divided into two groups: those diagnosed by emergency physician with ultrasound training and those diagnosed without training. The clinical characteristics and course for all patients were reviewed and compared for seeking the difference.

Results: A total of 182 children were included with the impression of intussuscpetion. One hundred seven (58.7\%) children diagnosed by pediatric emergency physician with ultrasound training and 75 diagnosed by those without ultrasound training. The gender, age, duration of symptoms, clinical symptoms were not statistically different between those two groups. The diagnostic sensitivity of the training group was significantly higher than the other group $(89.3 \%$ vs $76.9 \% \mathrm{p}=0.03)$. Children of the training group also had significantly shorter duration before enema reduction than the other group at emergency department $(2.28 \pm 1.55 \mathrm{~h}$ vs $4.78 \pm 4.99 \mathrm{~h}, \mathrm{p}=0.001)$.

Conclusion: Emergency physicians with ultrasound training has higher diagnostic sensitivity for pediatric intussusecption and spend fewer time before reduction at emergency department. 